\title{
Albumin-globulin ratio: a novel predictor of sepsis after flexible ureteroscopy in patients with solitary proximal ureteral stones
}

\author{
Junlin Lu\#, Yang Xun", Xiao Yu, Zheng Liu, Lei Cui, Jiaqiao Zhang, Cong Li, Shaogang Wang \\ Department of Urology, Tongji Hospital, Tongji Medical College, Huazhong University of Science and Technology, Wuhan, China \\ Contributions: (I) Conception and design: J Lu, Y Xun, C Li; (II) Administrative support: C Li, S Wang; (III) Provision of study materials or patients: \\ X Yu, Z Liu, L Cui, J Zhang; (IV) Collection and assembly of data: J Lu, Y Xun; (V) Data analysis and interpretation: J Lu; (VI) Manuscript writing: \\ All authors; (VII) Final approval of manuscript: All authors. \\ \#These authors contributed equally to this work. \\ Correspondence to: Cong Li; Shaogang Wang. Department of Urology, Tongji Hospital, Tongji Medical College, Huazhong University of Science and \\ Technology, Wuhan, China. Email: licongtjm@163.com; sgwangtjm@163.com.
}

Background: To identify predictors of sepsis after flexible ureteroscopy (fURS) in patients with unilateral,
solitary, proximal ureteral stones and evaluate the association between albumin-globulin ratio (AGR) and
sepsis.

Methods: Overall, 759 patients who underwent fURS were retrospectively enrolled. Univariate and multivariate logistic regression analyses were performed to identify predictors of sepsis after fURS in patients with solitary proximal ureteral stones. Then A nomogram was generated using the predictors.

Results: Sepsis occurred in 43 patients (5.7\%), and 6 (0.8\%) of them developed septic shock. Univariate analysis showed that age, female sex, albumin concentration, globulin concentration, AGR, pre-operative fever, white blood cell (WBC) count, urine culture (UC) result, urine WBC count, and urine nitrite result were predictors of sepsis. Multivariate analysis identified AGR $<1.2$ [odds ratio $(\mathrm{OR})=2.810,95 \%$ confidence interval (CI): $1.245-6.342, \mathrm{P}=0.013$ ] and positive $\mathrm{UC}(\mathrm{OR}=10.520,95 \% \mathrm{CI}: 4.489-24.653, \mathrm{P}<0.001)$ as independent predictors. When AGR $<1.2$ and positive $\mathrm{UC}$ were combined, area under the receiver operator characteristic curve was 0.825 . Then patients were categorized by different AGR level (" $\geq 1.4$ ", "1.2-1.4", “1.0-1.2", "<1.0"), and corresponding sepsis rates were $27.7 \%, 9.8 \%, 4.5 \%$ and $2.5 \%$.

Conclusions: Positive UC and low AGR were independent predictors of post-fURS sepsis. Cautious pre-operative evaluation and optimized treatment strategy should be considered to minimize infectious complications.

Keywords: Sepsis; flexible ureteroscopy (fURS); albumin globulin ratio (AGR); ureteral stone; positive urine culture (positive UC)

Submitted Mar 26, 2020. Accepted for publication Aug 28, 2020.

doi: 10.21037/tau-20-823

View this article at: http://dx.doi.org/10.21037/tau-20-823

\section{Introduction}

Flexible ureteroscopy (fURS) is recommended as first-line treatment for proximal ureteral stones $<20 \mathrm{~mm}$ because of its relatively high stone free rate and low complication rate $(1,2)$. Post-operative sepsis is the primary lethal complication with an incidence of $0.3-7.4 \%$, and it can lead to septic shock, organ failure, and death $(3,4)$.

Predictors are extensively identified to better understand
post-fURS sepsis, and they include stone height, stent indwelling time, and positive urine culture (UC) $(5,6)$. Most studies focused on urinary tract disorders: abnormal anatomy, stone characteristics or urinary tract infection (5-8). However, except for the condition of the urinary system, the systemic condition of patients is often underestimated with regard to post-fURS infections. Serum albumin concentration $<35 \mathrm{~g} / \mathrm{L}$ reportedly increases the risk of 
infection after orthopedic surgery by 2.5 times, and it is considered a malnutrition marker (9). Low serum albumin concentration can also predict infectious complications after cardiac surgery and oncologic surgery $(10,11)$. Globulin is an acute phase protein in host inflammatory immune response, and its concentration increases shortly after pathogen and toxin invasion (12). High globulin concentration indicates recent defensive response to infection. In this case, the operation may lead to the spread of inflammation. Albumin-globulin ratio (AGR) is a likely predictor of post-operative sepsis, but this has not been practically determined.

In this study, we aimed to identify the pre-operative independent predictors for sepsis in patients with solitary proximal ureteral stones who underwent fURS. The sepsis predictor profile will be clarified by both urinary and systemic condition. We present the following article in accordance with the STROBE reporting checklist (13) (available at http://dx.doi.org/10.21037/tau-20-823).

\section{Methods}

All procedures performed in this study were in accordance with the Declaration of Helsinki (as revised in 2013) and approved by the institutional research committee (Tongji Medical College, Huazhong University of Science and Technology, 2019\#S1035). Because of the retrospective nature of the research, the requirement for informed consent was waived. From January 2012 to December 2018, 759 patients who met the following inclusion criteria were enrolled: (I) history of fURS to treat unilateral, solitary, and proximal ureteral stones; (II) age $\geq 18$ years. The exclusion criteria were anatomical renal abnormalities such as transplant kidney, solitary kidney, horseshoe kidney, and kidney duplication.

Post-operative sepsis as defined by the 2001 International Sepsis Definitions Conference is the concurrence of infection and at least 2 of the following 4 criteria within 48 hours of surgery: (I) body temperature $>38{ }^{\circ} \mathrm{C}$; (II) heart rate $>90 /$ minute; (III) respiratory rate $>20 /$ minute; and (IV) leukocyte count $<4,000$ or $>12,000$ cells $/ \mu \mathrm{L}$ (14). Septic shock was defined as sepsis with persistent systolic arterial pressure $<90 \mathrm{mmHg}$ or systolic blood pressure reduction of $>40 \mathrm{mmHg}$ from baseline (14). The initial cutoff value of AGR was set at 1.2 based on the Youden index of the receiver operating characteristic (ROC) curves, which showed the highest sensitivity and specificity.

Proximal ureteral stones were confirmed by abdominal computed tomography, which was located above the fourth lumbar spine or in the ureteropelvic junction. Blood sample for liver function (including albumin and globulin) was routinely collected and analyzed after an overnight fast before surgery. Patients with indicators of infection, such as positive UC, fever, and leukocytosis, received at least a full antibiotic regimen (second-generation cephalosporins or levofloxacin) for 7 days until these infection indicators turned negative. Patients with no indicators of infection were administered a dose of prophylactic antibiotics. All the surgeries were performed by experienced urologists. If a double-J stent was inserted pre-operatively, it was removed at the beginning of surgery. Rigid ureteroscopy was routinely used for ureteral dilatation before fURS. A $0.035 \mathrm{~mm}$ guidewire was advanced through the urethral and ureteral meatuses to the renal pelvis under direct rigid ureteroscope vision. A 14-Fr ureteral access sheath (Cook Medical, Bloomington, IN) was then passed over the wire to the ureteropelvic junction, and 7.5-Fr fURS (Flex-X2, Karl Storz, Germany) was performed through the sheath. Intrarenal pressure was stabilized by a pressure-sensitive pump (Shenda Medical, China). Holmium: yttriumaluminum-garnet laser was used to fragment the stones. After lithotripsy, 6-Fr double-J stent was routinely inserted in all cases for 2 weeks.

The primary outcome was post-operative sepsis within 48 hours of surgery. Potential predictors were collected from our hospital's database, and they included the following: patient age and sex, body mass index (BMI), presence of comorbidities (hypertension, coronary heart disease, and diabetes mellitus), stone height, prior indwelling stent, hydronephrosis, cholesterol and creatinine concentrations, serum albumin and globulin concentrations, pre-operative fever (defined as body temperature $>38^{\circ} \mathrm{C}$ ), white blood cell (WBC) count, pre-operative UC, urine nitrite concentration, and urine $\mathrm{WBC}$ count (positive result refers to urine $\mathrm{WBC} \geq 50 / \mu \mathrm{L}$ in urine sediment analysis). All the factors closed to surgery were obtained. If the patients had preoperative infection, the infectious index (fever, UC, urine test, and urine $\mathrm{WBC}$ ) in the infectious episode were recorded.

\section{Statistical analysis}

Statistical analysis was performed using version 24.0 of the Statistical Package for Social Sciences software. The continuous variables had normal distributions as determined using Kolmogorov-Smirnov test, and they were compared 


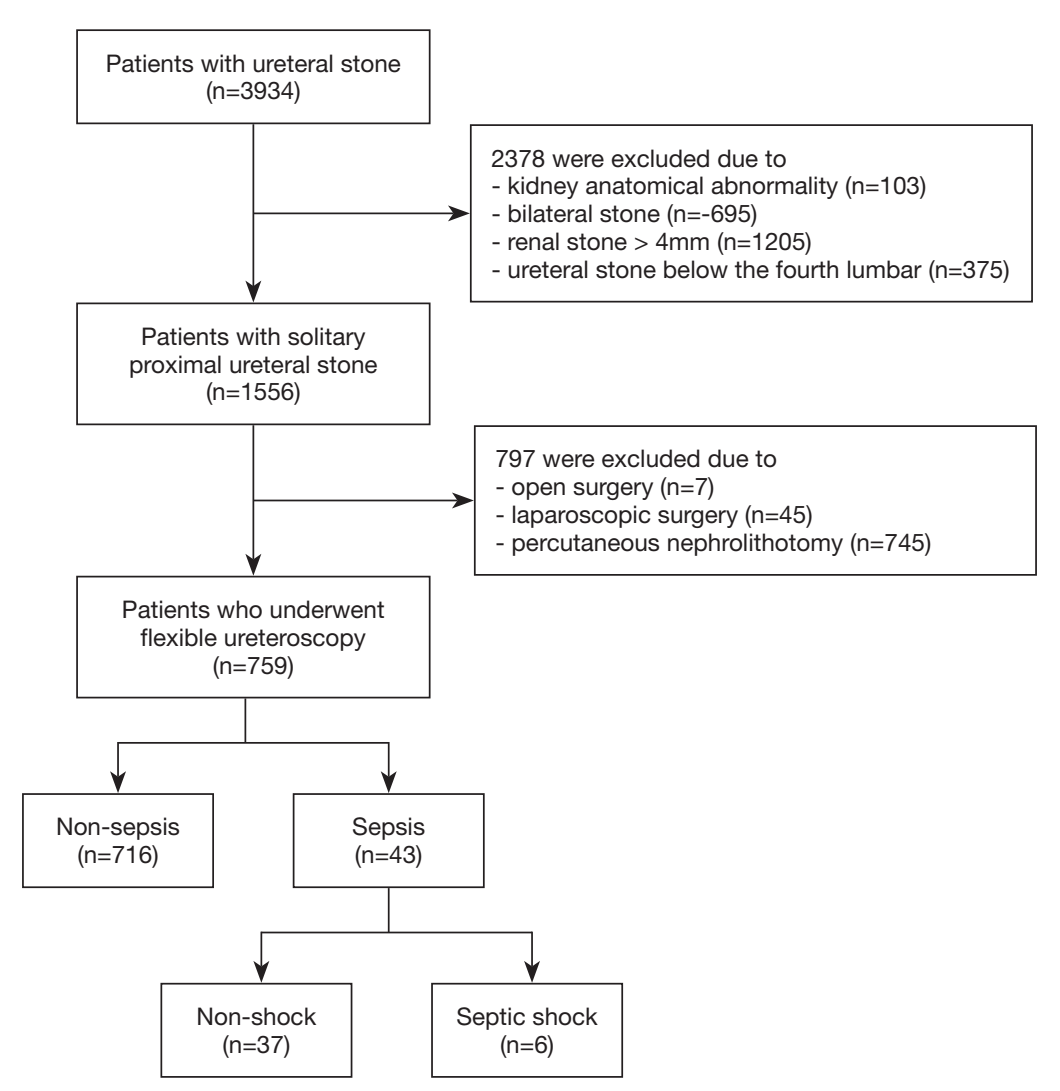

Figure 1 Patient selection flow diagram.

using Student's $t$-test. The chi-square test or Fisher's exact test was used to detect differences between categorical variables. Multivariate logistic regression analysis was used to identify independent predictors of post-operative sepsis. Bar diagrams and a nomogram prediction model for postoperative sepsis were created. Area under curve (AUC) was calculated from ROC curves to evaluate the superiority of these independent predictive factors of sepsis. Two-tailed $\mathrm{P}$ values $<0.05$ were considered statistically significant for all tests.

\section{Results}

\section{Independent predictors of sepsis after fURS}

Out of 3934 patients with ureteral stones, 759 underwent fURS and were selected (Figure 1). Of these 759 patients, $43(5.67 \%)$ developed sepsis and $6(0.79 \%)$ developed septic shock after fURS.

Patient characteristics and univariate analysis are presented in Table 1. There was a significant difference in age between patients who developed sepsis and patients who did not develop sepsis $(52.7 \pm 12.1$ versus $48.5 \pm 13.3$ years $)$. The percentage of women was significantly higher in patients who developed sepsis than in patients who did not develop sepsis $(\mathrm{P}<0.05)$. Patients who developed sepsis also had albumin concentrations $<35 \mathrm{~g} / \mathrm{L}$, globulin concentrations $\geq 30 \mathrm{~g} / \mathrm{L}, \mathrm{AGR}<1.2$, pre-operative fever, WBC count $\geq 10,000$ cells $/ \mu \mathrm{L}$, pre-operative positive $\mathrm{UC}$, positive urine $\mathrm{WBC}$, and positive urine nitrite. Factors with no significant effect on sepsis include BMI, operation time, stone height, stone laterality, indwelling catheter, hydronephrosis, hypertension, coronary heart disease, diabetes mellitus, cholesterol level, creatinine level, and ASA score.

Multivariate logistic analysis showed that AGR $<1.2$ and positive UC were independent predictors of sepsis with odds ratios (ORs) of 2.810 and 10.520 respectively (Table 2). Based on the predictors, the patients were classified into the following groups: positive $\mathrm{UC}$ with $\mathrm{AGR}<1.2$, positive $\mathrm{UC}$ with $\mathrm{AGR} \geq 1.2$, negative $\mathrm{UC}$ with $\mathrm{AGR} \geq 1.2$, and negative $\mathrm{UC}$ with AGR $<1.2$ (Figure 2). The corresponding sepsis rates of the 4 groups were $51.6 \%, 23.8 \%, 7.0 \%$, and $1.6 \%$. 
Table 1 Patients characteristics and univariate analysis of predictors for post-operative sepsis after flexible ureteroscopy

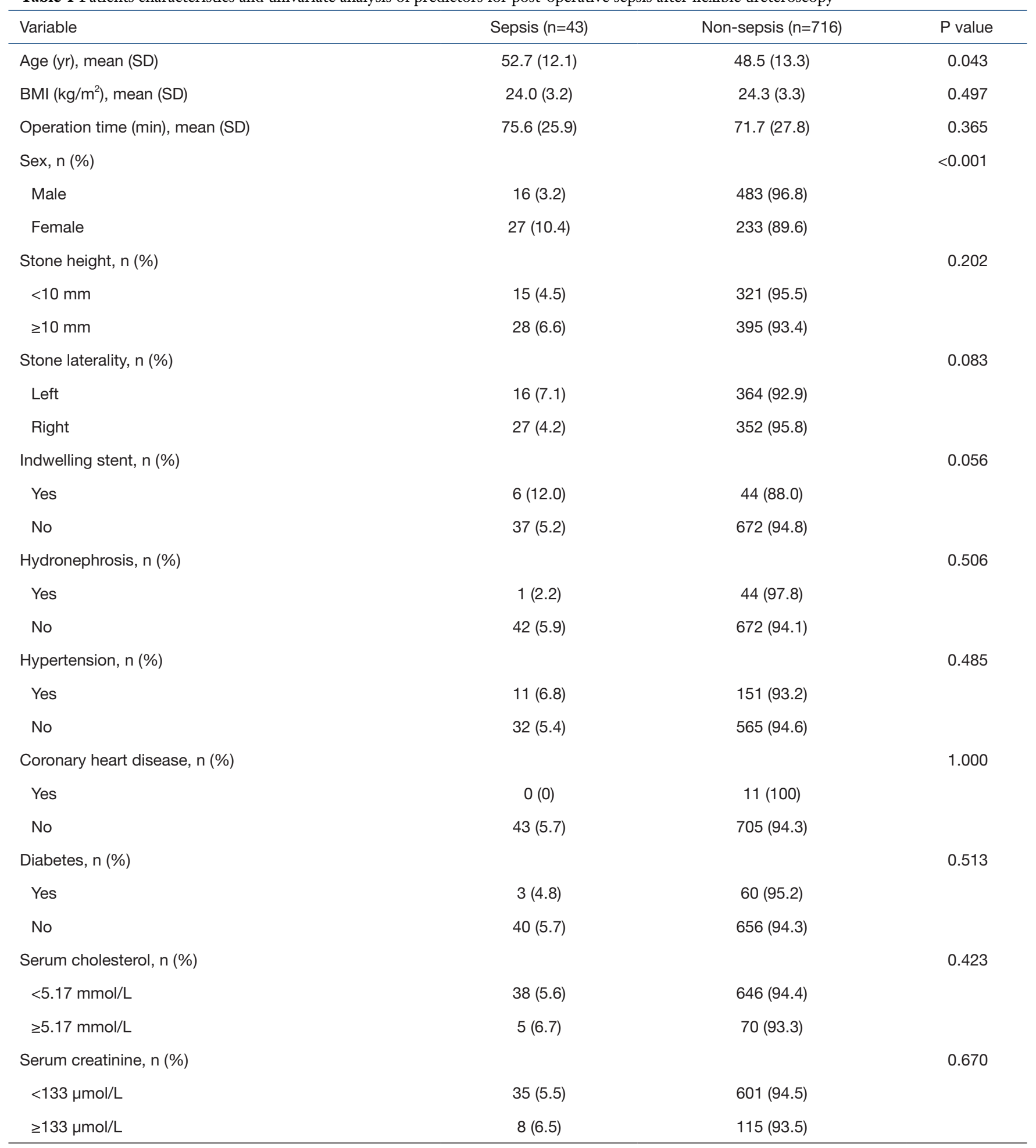

Table 1 (continued) 
Table 1 (continued)

\begin{tabular}{|c|c|c|c|}
\hline Variable & Sepsis $(n=43)$ & Non-sepsis $(n=716)$ & $P$ value \\
\hline Albumin, n (\%) & & & 0.001 \\
\hline$<35 \mathrm{~g} / \mathrm{L}$ & $12(15.6)$ & $65(84.4)$ & \\
\hline$\geq 35 \mathrm{~g} / \mathrm{L}$ & $31(4.5)$ & $651(95.5)$ & \\
\hline Globulin, n (\%) & & & 0.002 \\
\hline$<30 \mathrm{~g} / \mathrm{L}$ & $18(3.7)$ & $465(96.3)$ & \\
\hline$\geq 30 \mathrm{~g} / \mathrm{L}$ & $25(9.1)$ & $251(90.9)$ & \\
\hline AGR, n (\%) & & & $<0.001$ \\
\hline$<1.2$ & $24(15.1)$ & $135(84.9)$ & \\
\hline$\geq 1.2$ & $19(3.2)$ & $581(96.8)$ & \\
\hline Pre-operative fever, n (\%) & & & 0.001 \\
\hline Yes & $8(21.1)$ & $30(78.9)$ & \\
\hline No & $35(4.9)$ & $686(95.1)$ & \\
\hline WBC, n (\%) & & & 0.005 \\
\hline$<10,000$ cells $/ \mu \mathrm{L}$ & $33(4.8)$ & $654(95.2)$ & \\
\hline$\geq 10,000 \mathrm{cells} / \mu \mathrm{L}$ & $10(13.9)$ & $62(86.1)$ & \\
\hline Urine culture, n (\%) & & & $<0.001$ \\
\hline Positive & $25(34.2)$ & $48(65.8)$ & \\
\hline Negative & $18(2.6)$ & $668(97.4)$ & \\
\hline Urine WBC, n (\%) & & & $<0.001$ \\
\hline Positive & $31(9.1)$ & $309(90.9)$ & \\
\hline Negative & $12(2.9)$ & $407(97.1)$ & \\
\hline Urine nitrite, n (\%) & & & $<0.001$ \\
\hline Positive & $13(37.1)$ & $22(62.9)$ & \\
\hline Negative & $30(4.1)$ & $694(95.9)$ & \\
\hline
\end{tabular}

BMI, body mass index; SD, standard deviation; AGR, albumin globulin ratio; WBC, white blood cell.

Patients with positive UC and AGR $<1.2$ had a 23.4-fold risk of developing sepsis than the other patients [OR: 23.4, $95 \%$ confidence interval $(\mathrm{CI}): 10.5-52.1 ; \mathrm{P}<0.001]$.

Six patients developed post-operative septic shock and their clinical characteristics are shown in Table 3. Their mean AGR was 1.11 and $66.7 \%$ of them had positive UC. In contrast, $58.1 \%$ of patients who developed sepsis had positive UCs and a mean AGR of 1.16, and the corresponding values for patients who did not develop sepsis were $6.7 \%$ and 1.42 . A similar trend was also observed with female sex, pre-operative fever, hypertension, indwelling stent, hydronephrosis, WBC count, urine WBC count, and urine nitrite concentration.

\section{Predictor evaluation and prediction model creation}

The combined predictor was calculated based on a prior multivariate analysis. The AUC values for the combined factor, positive UC, and AGR were 0.825, 0.757, and 0.685 respectively (Figure 3). Further analysis was conducted, which divided AGR into 4 categories and determined the sepsis rate at different AGR levels. The sepsis rates were $27.7 \%, 9.8 \%, 4.5 \%$, and $2.5 \%$ at AGR values $<1.0$, $1.0-1.2,1.2-1.4$, and $\geq 1.4$ respectively (Figure $4 A$ ). Thus, a 
Table 2 Multivariate logistic regression analysis of predictors of sepsis after flexible ureteroscopy

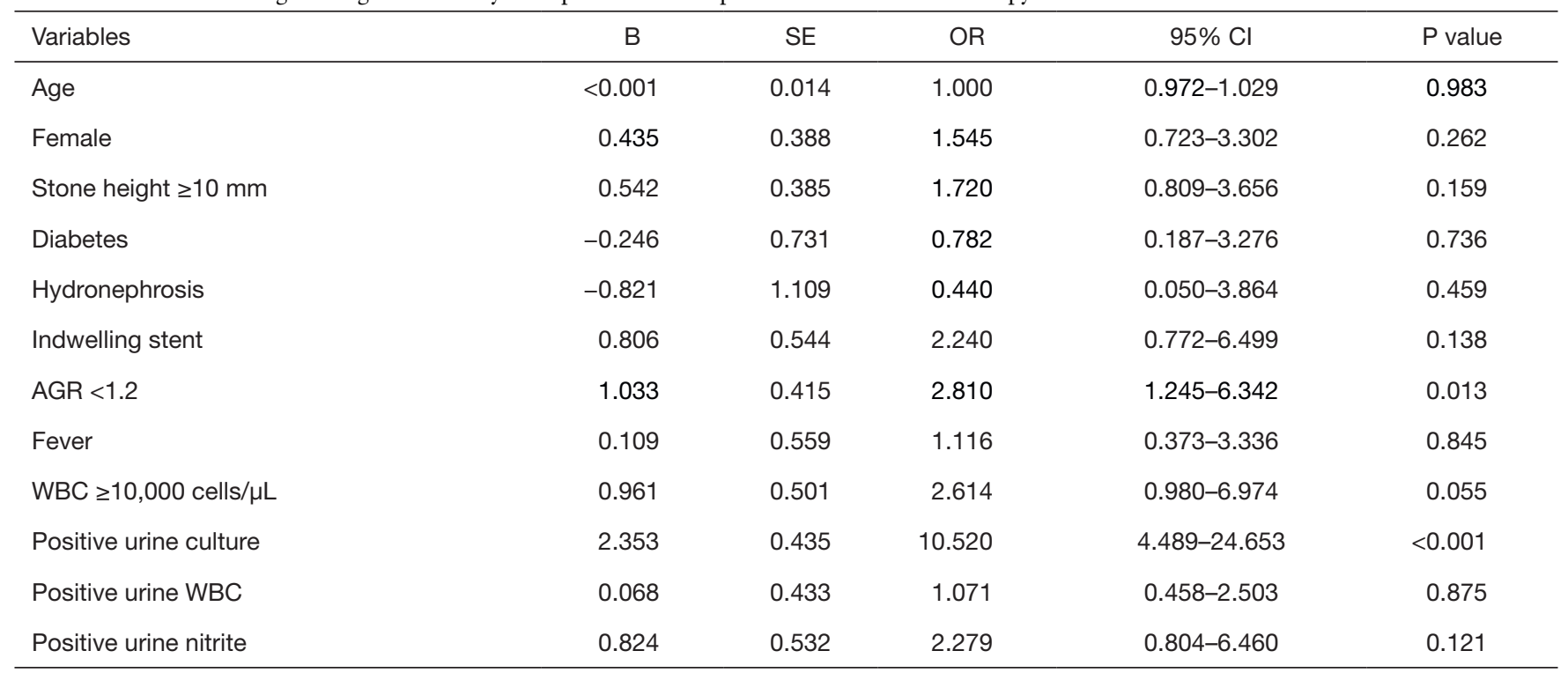

AGR, albumin globulin ratio; WBC, white blood cell; B, regression coefficient; SE, standard error; OR, odds ratio; Cl, confidence interval.

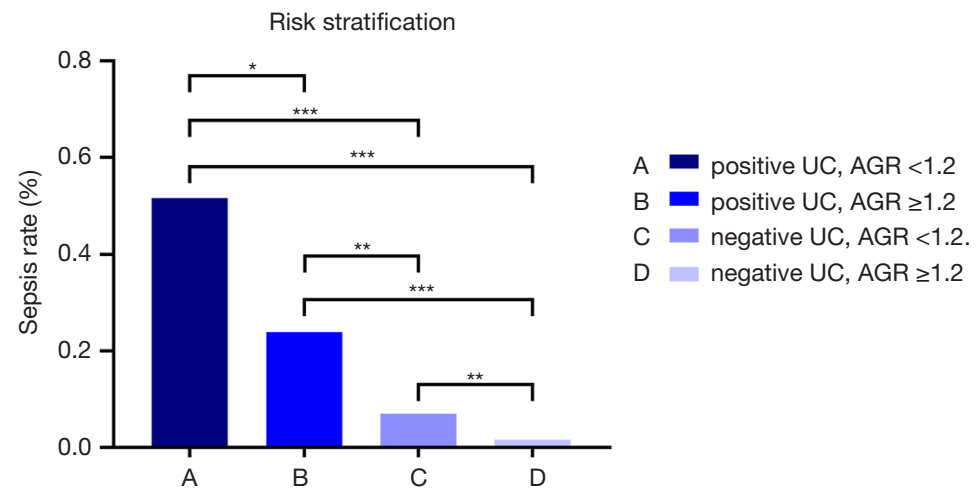

Figure 2 Sepsis rate for different patient group stratified by UC and AGR. ${ }^{*} \mathrm{P}<0.05 ;{ }^{* *} \mathrm{P}<0.01 ;{ }^{* * *} \mathrm{P}<0.001$. UC, urine culture; AGR, albumin globulin ratio.

nomogram prediction model was constructed to calculate the cumulative probability of post-operative sepsis. Points were obtained for a variable by tracing a line upwards from the variable axis, and another line is traced downwards to the total points axis, which sums up all the points on the sepsis axis, and the cumulative probability of post-operative sepsis is thereby determined (Figure $4 B$ ).

\section{Discussion}

Sepsis after fURS is a life-threatening complication that substantially increases hospital stay, mortality risk, and economic burden on patients and the nation (3). We found that the sepsis rate after using fURS to treat patients with proximal ureteral stones was $5.67 \%$. Our result was consistent with those of earlier studies $(4,15)$. Among all the pre-operative predictors, positive UC and AGR $<1.2$ were identified as independent predictors.

Patients with positive UC, despite administration of a full course of antibiotics and conversion to a negative result, had a 10.52-fold risk of sepsis after fURS, which is similar to the findings of a previous study. Uchida (7) and Blackmur (4) reported that positive bladder UC is an independent predictor of infectious complications that 
Table 3 Characteristics of 6 patients with post-fURS septic shock (patient named by A to F)

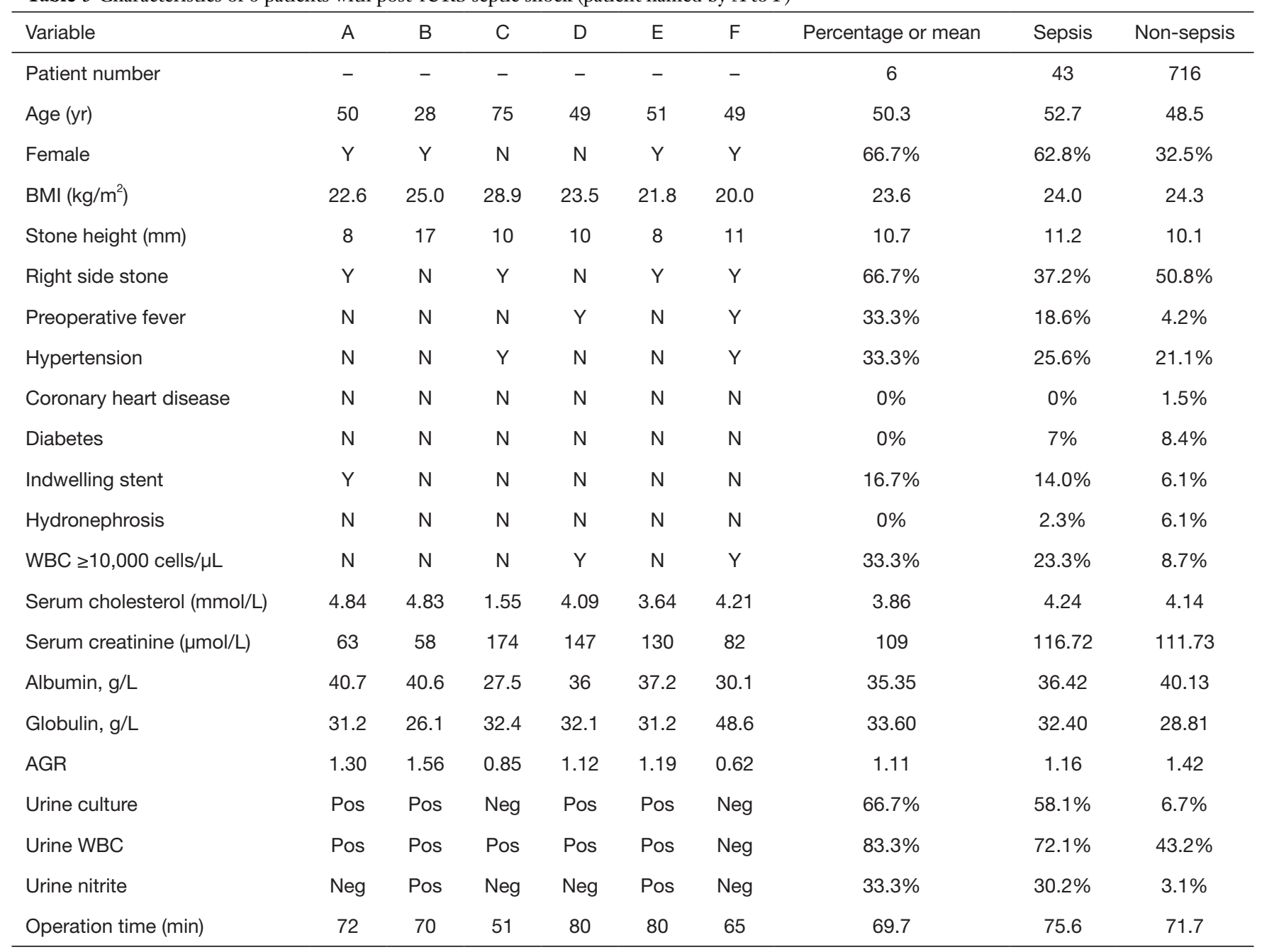

fURS, flexible ureteroscopy; BMI, body mass index; WBC, white blood cell; AGR, albumin

increases risk 3.52-4.88 times. This may be because high intrarenal pressure during fURS promotes invasion of blood circulation by local pathogens and toxins. Nevertheless, when stone culture (8), stent culture (15) or pelvic UC (16) was analyzed with bladder UC in multivariate analysis, no significant differences were found between bladder UC and post-fURS infection, but sepsis rate increased significantly with positive stone culture, stent culture, or pelvic UC. The results highlight the key role of the microbial environment of the kidney, bladder, and stone in the development of infection. However, samples cannot be obtained preoperatively from the kidney, stone, or stent, and this limits the determination of the role of the microbial environment in early prediction and prevention. Mid-stream bladder UC is still a recognized optimal predictor that can be acquired early.

This study also revealed that low AGR $(<1.2)$ leads to a 2.81 -fold increase in the risk of post-fURS sepsis. Low AGR is mainly used as a predictor of cancer progress and cancerrelated mortality due to its reflection of the state of human nutrition, inflammation, and immunity (17). Nutritional state is an important aspect of the pre-operative state, and it includes weight, BMI, and serum albumin concentration. Rapp-Kesek (10) investigated 886 patients who underwent cardiac surgery and found that albumin concentration has a negative correlation with post-operative infection $(\mathrm{OR}=0.04)$ but not with mortality. Albumin can play an indirect role in immunity by supplying nitrogen for globulin synthesis, 
by acting as a protective colloid to stabilize lyophobic proteins by surrounding them and by enabling globulin to clear pathogens and toxins. A meta-analysis confirmed that albumin concentration can predict infection after orthopedic surgery by showing that albumin concentrations

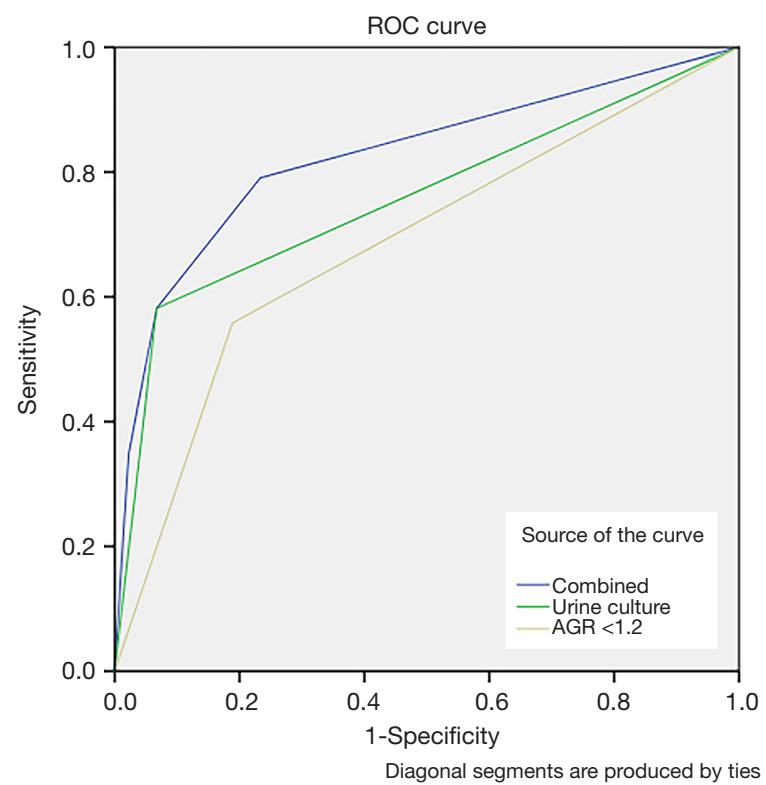

Figure 3 Receiver operator characteristic (ROC) curves for urine culture, AGR <1.2 and combined predictor. (Area under curve: 0.757 for urine culture, 0.685 for AGR $<1.2,0.825$ for combined predictor). AGR, albumin globulin ratio.
$<35 \mathrm{~g} / \mathrm{L}$ increases the risk of post-operative infection 2.39 times (9). In urinary stone surgery, Yang et al. (18) identified the risk factors of infectious complications after PCNL. They found that low albumin concentration, as well as stone size and urine WBC, was associated with post-PCNL fever. Studies on the relationship between serum globulin concentration and post-operative infection are scarce. In the early phase of infection, serum immunoglobulin level rapidly rises and presents as elevated globulin concentration $(12,19)$. Endourological surgery may facilitate backflow of pathogens and toxins, leading to spread of infection. In this study, univariate analysis revealed a significant association between high globulin concentration $(>30 \mathrm{~g} / \mathrm{L})$, low albumin concentration $(<35 \mathrm{~g} / \mathrm{L})$, and post-fURS sepsis. After the creation of a multivariate model, it was found that AGR is independently associated with sepsis. Considering that AGR can infer systemic state from nutritional state, inflammation, and immune state, we posit that AGR is a predictor of infection after endourological surgeries and infection-prone surgeries and time-consuming surgeries.

Interestingly, of the 6 patients with septic shock, 4 had positive pre-operative UC and 2 had negative pre-operative $\mathrm{UC}$ with very low AGRs of 0.85 and 0.62 . The patient with an AGR of 0.85 was 75 years old and had a serum albumin concentration of $27.5 \mathrm{~g} / \mathrm{L}$, showing a poor nutritional state. The patient with an AGR of 0.62 had pre-operative fever, leukocytosis, and a serum globulin concentration of $48.6 \mathrm{~g} / \mathrm{L}$, indicating an episode of apparent infection. In
A

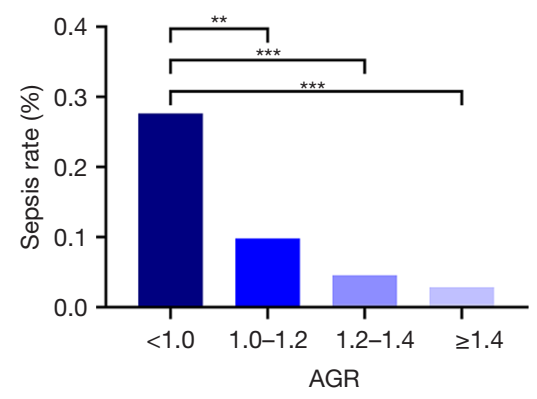

B

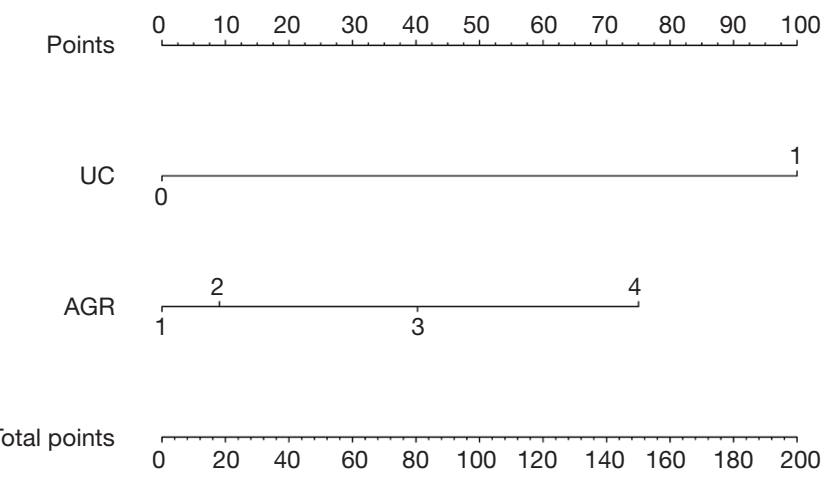

SEPSIS

$$
\begin{array}{lllllll}
0.1 & 0.2 & 0.3 & 0.4 & 0.5 & 0.6 & 0.7
\end{array}
$$

Figure 4 (A) Categorized albumin globulin ratio (AGR) and corresponding sepsis rate; (B) Nomogram for patients predicting post-operative sepsis. "1" refers to positive and "0" refers to negative. AGR: "1" to "4" refers to " $\geq 1.4$ ", "1.2-1.4", "1.0-1.2", "<1.0" respectively. ** $\mathrm{P}<0.01$; *** $\mathrm{P}<0.001$. UC, urine culture. 
analyzing the categories of AGR, it was found that sepsis rate is higher when AGR is lower. The results reemphasized the importance of UC and AGR.

There are many studies on infectious risk factors of endourological procedures, which are divided into pre, intra-, and post-procedure risk factors. However, most studies focused on the factor itself. A "good" factor should have high sensitivity and specificity, and it should guide early evaluation and intervention to "nip it in the bud". Based on large-population evidence, we proposed a urologic predictor (positive UC) and a systemic predictor (low AGR) of post-fURS sepsis. New devices that reduce intrarenal pressure or improve irrigation may produce similar results, and these require further research.

The main limitation of this study is its retrospective nature. Patient information was retrieved from the medical records system of a tertiary hospital. We did not analyze stone imaging data in detail because some imaging results were obtained from other hospitals. This is the first study on solitary proximal ureteral stones and infectious complications in a large number of patients. Stone location, size, and burden were within a narrow range, which may have a similarly narrow range of effects on sepsis. In this study, we only enrolled patients with proximal ureteral stone instead of other stone types (renal stone or distal ureteral stone). Proximal ureteral stones are relatively uniform in size, location, and burden, while renal stones are heterogeneous that may have an influence on sepsis rate after fURS.

\section{Conclusions}

Patients with solitary proximal ureteral stones are more likely to have post-fURS sepsis when they have positive pre-operative UC or low pre-operative AGR. With the categorization of AGR, it was evident that the lower the AGR, the higher the sepsis rate. Early evaluation of patient urologic and systemic states can be useful in treatment strategy optimization. Attention should be paid to the nutritional and inflammatory state of patients. Further studies are necessary to better understand and prevent infectious complications after urinary stone surgery.

\section{Acknowledgments}

Funding: This research is supported by grants from the National Natural Science Foundation of China (NSFC, 81974092).

\section{Footnote}

Reporting Checklist: The authors have completed the STROBE reporting checklist. Available at http://dx.doi. org/10.21037/tau-20-823

Data Sharing Statement: Available at http://dx.doi. org/10.21037/tau-20-823

Peer Review File: Available at http://dx.doi.org/10.21037/ tau-20-823

Conflicts of Interest: All authors have completed the ICMJE uniform disclosure form (available at http://dx.doi. org/10.21037/tau-20-823). The authors have no conflicts of interest to declare.

Ethical Statement: The authors are accountable for all aspects of the work in ensuring that questions related to the accuracy or integrity of any part of the work are appropriately investigated and resolved. All procedures performed in this study were in accordance with the Declaration of Helsinki (as revised in 2013) and approved by the institutional research committee (Tongji Medical College, Huazhong University of Science and Technology, 2019\#S1035). Because of the retrospective nature of the research, the requirement for informed consent was waived.

Open Access Statement: This is an Open Access article distributed in accordance with the Creative Commons Attribution-NonCommercial-NoDerivs 4.0 International License (CC BY-NC-ND 4.0), which permits the noncommercial replication and distribution of the article with the strict proviso that no changes or edits are made and the original work is properly cited (including links to both the formal publication through the relevant DOI and the license). See: https://creativecommons.org/licenses/by-nc-nd/4.0/.

\section{References}

1. Babjuk. M, Burger. M, Compérat. E, et al. EAU Guidelines. Edn. presented at the EAU Annual Congress Amsterdam 2020. ISBN 978-94-92671-07-3. 2020; EAU Guidelines Office, Arnhem, The Netherlands. Available online: http://uroweb.org/guidelines/compilations-of-allguidelines/

2. Legemate JD, Wijnstok NJ, Matsuda T, et al. Characteristics and outcomes of ureteroscopic treatment 
in 2650 patients with impacted ureteral stones. World J Urol 2017;35:1497-506.

3. Somani BK, Giusti G, Sun Y, et al. Complications associated with ureterorenoscopy (URS) related to treatment of urolithiasis: the Clinical Research Office of Endourological Society URS Global study. World J Urol 2017;35:675-81.

4. Blackmur JP, Maitra NU, Marri RR, et al. Analysis of Factors' Association with Risk of Postoperative Urosepsis in Patients Undergoing Ureteroscopy for Treatment of Stone Disease. J Endourol 2016;30:963-9.

5. Nevo A, Mano R, Baniel J, et al. Ureteric stent dwelling time: a risk factor for post-ureteroscopy sepsis. BJU Int 2017;120:117-22.

6. Sohn DW, Kim SW, Hong CG, et al. Risk factors of infectious complication after ureteroscopic procedures of the upper urinary tract. J Infect Chemother 2013;19:1102-8.

7. Uchida Y, Takazawa R, Kitayama S, et al. Predictive risk factors for systemic inflammatory response syndrome following ureteroscopic laser lithotripsy. Urolithiasis 2018;46:375-81.

8. Eswara JR, Shariftabrizi A, Sacco D. Positive stone culture is associated with a higher rate of sepsis after endourological procedures. Urolithiasis 2013;41:411-4.

9. Yuwen $\mathrm{P}, \mathrm{Chen} \mathrm{W}, \mathrm{Lv} \mathrm{H}$, et al. Albumin and surgical site infection risk in orthopaedics: a meta-analysis. BMC Surg 2017;17:7.

10. Rapp-Kesek D, Ståhle E, Karlsson TT. Body mass index and albumin in the preoperative evaluation of cardiac surgery patients. Clin Nutr 2004;23:1398-404.

11. Antoun S, Rey A, Béal J, et al. Nutritional risk factors in planned oncologic surgery: what clinical and biological

Cite this article as: Lu J, Xun Y, Yu X, Liu Z, Cui L, Zhang J, Li C, Wang S. Albumin-globulin ratio: a novel predictor of sepsis after flexible ureteroscopy in patients with solitary proximal ureteral stones. Transl Androl Urol 2020;9(5):1980-1989. doi:10.21037/tau-20-823 parameters should be routinely used? World J Surg 2009;33:1633-40.

12. Busani S, Damiani E, Cavazzuti I, et al. Intravenous immunoglobulin in septic shock: review of the mechanisms of action and meta-analysis of the clinical effectiveness. Minerva Anestesiol 2016;82:559-72.

13. von Elm E, Altman DG, Egger M, et al. The Strengthening the Reporting of Observational Studies in Epidemiology (STROBE) statement: guidelines for reporting observational studies. Lancet 2007;370:1453-7.

14. Levy MM, Fink MP, Marshall JC, et al. 2001 SCCM/ ESICM/ACCP/ATS/SIS International Sepsis Definitions Conference. Crit Care Med 2003;31:1250-6.

15. Nevo A, Mano R, Schreter E, et al. Clinical Implications of Stent Culture in Patients with Indwelling Ureteral Stents Prior to Ureteroscopy. J Urol 2017;198:116-21.

16. Yoshida S, Takazawa R, Uchida Y, et al. The significance of intraoperative renal pelvic urine and stone cultures for patients at a high risk of post-ureteroscopy systemic inflammatory response syndrome. Urolithiasis 2019;47:533-40.

17. Lv GY, An L, Sun XD, et al. Pretreatment albumin to globulin ratio can serve as a prognostic marker in human cancers: a meta-analysis. Clin Chim Acta 2018;476:81-91.

18. Yang T, Liu S, Hu J, et al. The Evaluation of Risk Factors for Postoperative Infectious Complications after Percutaneous Nephrolithotomy. Biomed Res Int 2017;2017:4832051.

19. Davis CP, Cohen MS, Anderson MD, et al. Total and specific immunoglobulin response to acute and chronic urinary tract infections in a rat model. J Urol 1987;138:1308-17. 